\title{
Article
}

\section{The migration of gas giant planets in gravitationally unstable disks}

Stamatellos, Dimitris

Available at https://clok.uclan.ac.uk/12676/

Stamatellos, Dimitris orcid iconORCID: 0000-0002-4502-8344 (2015) The migration of gas giant planets in gravitationally unstable disks. The Astrophysical Journal Letters, 810 . L11. ISSN 2041-8205

It is advisable to refer to the publisher's version if you intend to cite from the work. http://dx.doi.org/10.1088/2041-8205/810/1/L11

For more information about UCLan's research in this area go to http://www.uclan.ac.uk/researchgroups/ and search for < name of research Group>.

For information about Research generally at UCLan please go to http://www.uclan.ac.uk/research/

All outputs in CLoK are protected by Intellectual Property Rights law, including Copyright law. Copyright, IPR and Moral Rights for the works on this site are retained by the individual authors and/or other copyright owners. Terms and conditions for use of this material are defined in the policies page.

\section{CLoK}

Central Lancashire online Knowledge www.clok.uclan.ac.uk

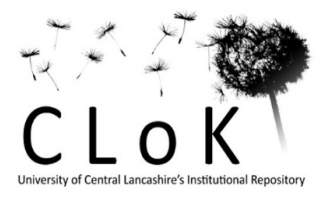




\title{
THE MIGRATION OF GAS GIANT PLANETS IN GRAVITATIONALLY UNSTABLE DISKS
}

\author{
Dimitris Stamatellos \\ Jeremiah Horrocks Institute for Mathematics, Physics \& Astronomy, University of Central Lancashire, Preston PR1 2HE, UK; dstamatellos@uclan.ac.uk \\ Received 2015 June 26; accepted 2015 July 31; published 2015 August 25
}

\begin{abstract}
Planets form in the disks of gas and dust that surround young stars. It is not known whether or not gas giant planets on wide orbits form the same way as Jupiter or form by the fragmentation of gravitationally unstable disks. Here we show that a giant planet that has formed in the outer regions of a protostellar disk initially migrates quickly toward the central star (migration timescale $\sim 10^{4}$ years) while accreting gas from the disk. However, in contrast with previous studies, we find that the planet eventually opens up a gap in the disk and the migration is essentially halted. At the same time, accretion-powered radiative feedback from the planet significantly limits its mass growth, keeping it within the planetary-mass regime, (i.e., below the deuterium burning limit) at least for the initial stages of disk evolution. Giant planets may therefore be able to survive on wide orbits despite their initial fast inward migration, consequently shaping the environment in which terrestrial planets that may harbor life can form.
\end{abstract}

Key words: hydrodynamics - planet-disk interactions - planets and satellites: formation - planets and satellites: gaseous planets - protoplanetary disks

\section{INTRODUCTION}

Observations of exoplanetary systems are important, as they provide significant clues about the formation of our solar system. In the last few years direct imaging of massive giant planets on wide orbits has become possible (Marois et al. 2008; Ireland et al. 2011; Aller et al. 2013; Kuzuhara et al. 2013; Rameau et al. 2013; Bailey et al. 2014; Galicher et al. 2014; Kraus et al. 2014). These planets challenge our understanding of planet formation, as they are unlikely to form by core accretion, i.e., the coagulation of dust particles to progressively larger aggregates and an accretion of a gaseous envelope (Safronov \& Zvjagina 1969; Goldreich \& Ward 1973; Mizuno 1980; Pollack et al. 1996). Gravitational fragmentation of protostellar disks is an alternative formation scenario (Kuiper 1951; Cameron 1978; Boss 1997; Boley 2009). It has been argued that protoplanets forming in gravitationally unstable disks quickly migrate toward the host star (Baruteau et al. 2011; Michael et al. 2011), where they may be disrupted by tidal torques (Nayakshin \& Cha 2013; Tsukamoto et al. 2013). Moreover, it has been suggested that protoplanets rapidly grow in mass by accreting material from the disk to become brown dwarfs and low-mass stars (Rafikov 2005; Stamatellos \& Whitworth 2009a; Zhu et al. 2012).

In this Letter we present models of the interaction of a newly formed massive planetary embryo (henceforth protoplanet) with a protostellar disk that contradict the above claims. Critically, we include in our models two physical ingredients whose combined effect has been ignored by previous studies: (i) gas accretion onto the protoplanet, and (ii) accretionpowered radiative feedback from the protoplanet.

\section{COMPUTATIONAL MODEL}

We assume that the protoplanet has formed in the protostellar disk of a Sun-like star by gravitational fragmentation. For this to happen the disk has to be relatively massive and the protoplanet's initial orbital radius is at least 50-100 AU, as these conditions are favorable for fragmentation only in the disk outer region (Rafikov 2005; Stamatellos \&
Whitworth 2009a). The minimum initial mass of the protoplanet is set by the opacity limit for fragmentation, which is thought to be $1-5 M_{\mathrm{J}}$, where $M_{\mathrm{J}}$ is the mass of Jupiter (Low \& Lynden-Bell 1976; Whitworth \& Stamatellos 2006; Boley et al. 2010; Kratter et al. 2010). Here we examine the evolution of such a protoplanet as it interacts with its parent disk, using a three-dimensional (3D) smoothed particle hydrodynamics (SPH) method that includes the effects of radiative transport in the disk (Stamatellos et al. 2007; Stamatellos \& Whitworth 2009b).

\subsection{Initial Conditions}

We assume a star-disk system in which the central star has an initial mass $M_{\star}=1 M_{\odot}$. The initial disk mass is $M_{\mathrm{D}}=0.1 M_{\odot}$ and the initial disk radius is $R_{\mathrm{D}}=100 \mathrm{AU}$. The disk is represented by $10^{6} \mathrm{SPH}$ particles. The inner disk boundary is set at $0.2 \mathrm{AU}$. The disk initial surface density is

$$
\Sigma_{0}(R)=\Sigma(1 \mathrm{AU})\left(\frac{R}{\mathrm{AU}}\right)^{-1},
$$

and the disk temperature is

$$
T_{0}(R)=250 \mathrm{~K}\left(\frac{R}{\mathrm{AU}}\right)^{-3 / 4}+10 \mathrm{~K}
$$

where $\Sigma(1 \mathrm{AU})$ is determined by the disk mass and radius, and $R$ is the distance from the central star measured on the disk midplane. The initial temperature profile corresponds to a flat disk that reprocesses stellar radiation and it is steeper when compared with observations (e.g., Andrews et al. 2009 find a temperature profile $R^{-q} ; q \approx 0.4-0.74$, for disks in Ophiuchus). However, the actual temperature profile attained once the disk is allowed to evolve is less steep due to additional heating sources (viscous and accretion heating).

We let the disk relax for $3 \mathrm{kyr}$ ( $\sim 3$ outer orbital periods) and we then embed in it a protoplanet of initial mass $M_{\mathrm{p}, \mathrm{i}}=1 M_{\mathrm{J}}$ at a distance $R_{\mathrm{p}, \mathrm{i}}=50 \mathrm{AU}$ from the central star and let the diskplanet system evolve. The protoplanet's initial velocity is set as 


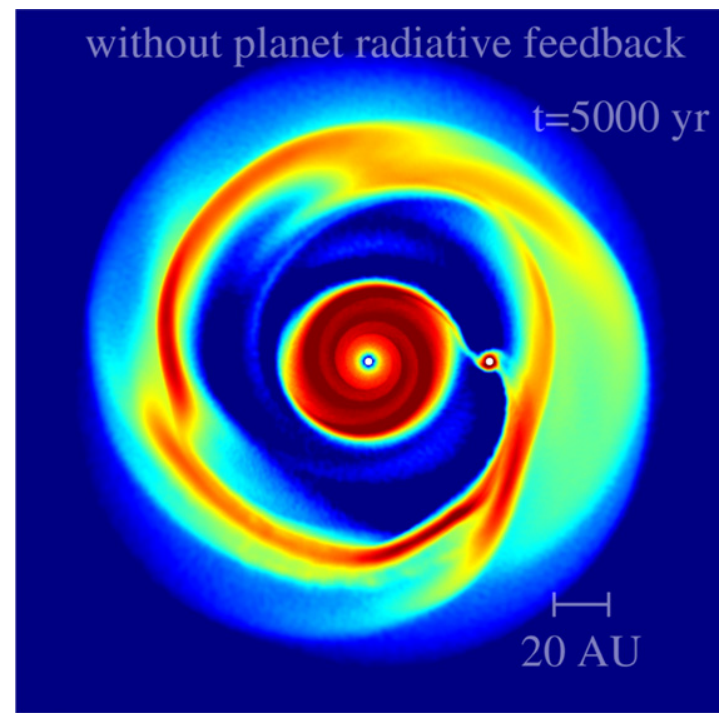

with planet radiative feedback

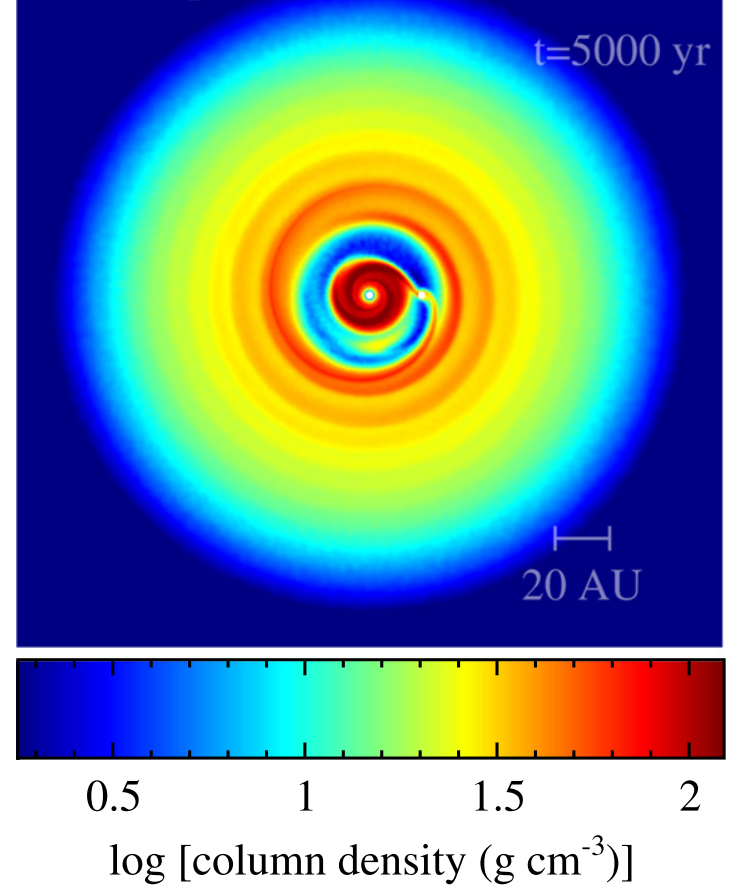

Figure 1. Column density plots showing gap opening induced by a Jupitermass protoplanet in an $0.1-M_{\odot}$ disk, in two simulations: without (top) and with (bottom) radiative feedback from the protoplanet. The star (in the center) and the protoplanet are depicted by thick white dots. The protoplanet in the first simulation opens up a deep, wide gap and grows to become a brown dwarf, migrating initially inward and subsequently outward. In the simulation with radiative feedback, the gap is shallow and narrow. The protoplanet migrates inward but its mass growth is suppressed, so it becomes a wide-orbit planet.

the same as the velocity of the local gas, i.e., Keplerian (including the contribution from the disk mass within the protoplanet's orbit). We assume an initial circular orbit (eccentricity $e_{\mathrm{i}}=0$ ). The protoplanet is allowed to accrete gas from the disk, if this gas approaches within $0.1 \mathrm{AU}$ from the protoplanet and is bound to it. This distance is always much smaller than the Hill radius of the protoplanet, which is defined as the region where the protoplanet's gravity dominates over the gravity of the central star. Therefore, the region around the protoplanet is resolved appropriately.

\subsection{Hydrodynamics and Radiative Transfer}

We use the SPH code SEREN (Hubber et al. 2011) to treat the gas thermodynamics. The code invokes an octal tree (to compute gravity and find neighbors), multiple particle time steps, and a second-order Runge-Kutta integration scheme. The disk self-gravity is therefore included in the simulations. The code uses time-dependent artificial viscosity with parameters $\alpha_{\min }=0.1, \alpha_{\max }=1$, and $\beta=2 \alpha$, so as to reduce artificial shear viscosity. The chemical and radiative processes that regulate the gas temperature are treated with the approximation of Stamatellos et al. (2007; see also Forgan et al. 2009). We adopt opacity tables that are appropriate for protostellar disks (Semenov et al. 2003).

\subsection{Star and Protoplanet Representation}

The central star and the protoplanet are represented by sink particles that interact with the rest of the computational domain only through their gravity (and luminosity, when irradiation from the protoplanet is taken into account). The sink radius of the central star is set to $R_{\mathrm{sink}, \star}=0.2 \mathrm{AU}$, and the sink radius of the protoplanet is set to $R_{\text {sink,p }}=0.1 \mathrm{AU}$. This value is always much smaller (by at least a factor of $\sim 25$ ) than the Hill radius of the protoplanet, which defined as the region where the gravity of the protoplanet dominates over the gravity of the star, i.e., $R_{\text {sink, } \mathrm{p}}<R_{\mathrm{H}}=R\left(M_{\mathrm{p}} / 3 M_{\star}\right)^{1 / 3}$. The Hill radius increases as the protoplanet accretes material from the disk or it decreases as the protoplanet moves closer to the central star. Gas particles accrete onto a star or protoplanet sink when they are within the sink radius and bound to the sink.

\subsection{Radiative Feedback from the Star and the Protoplanet}

The radiation feedback from the star and the protoplanet is taken into account by invoking a pseudo-ambient radiation field with a temperature $T_{\mathrm{A}}(\boldsymbol{r})$ that is a function of the position relative to the star and the protoplanet (Stamatellos et al. 2007, 2011, 2012). This temperature effectively sets the minimum temperature that the gas can attain when cooled radiatively. The contribution to $T_{\mathrm{A}}(\boldsymbol{r})$ from the central star is set to

$$
T_{\mathrm{A}}^{\star}(\boldsymbol{r})=250 \mathrm{~K}\left(\frac{R}{\mathrm{AU}}\right)^{-3 / 4}+10 \mathrm{~K},
$$

where $R$ is the distance from the star measured on the disk midplane. The contribution to $T_{\mathrm{A}}(\boldsymbol{r})$ from the protoplanet is

$$
T_{\mathrm{A}}^{\text {planet }}(\boldsymbol{r})=\left(\frac{L_{\mathrm{p}}}{16 \pi \sigma_{\mathrm{SB}}\left|\boldsymbol{r}-\boldsymbol{r}_{\mathrm{p}}\right|^{2}}\right)^{1 / 4},
$$

where $L_{\mathrm{p}}$ and $\boldsymbol{r}_{\mathrm{p}}$, are the luminosity and position of the protoplanet, respectively. This luminosity is given by

$$
L_{\mathrm{p}}=f \frac{G M_{\mathrm{p}} \dot{M}_{\mathrm{p}}}{R_{\mathrm{acc}}},
$$

where $M_{\mathrm{p}}$ is the mass of the protoplanet, $\dot{M}_{\mathrm{p}}$ is the accretion rate onto it, and $R_{\text {acc }}$ is the accretion radius. $f=0.75$ is the fraction of the accretion energy that is radiated away at the surface of the protoplanet rather than being expended via driving jets and/or winds (Machida et al. 2006). As we assume that this planet has formed by gravitational instabilities in the disk, the 

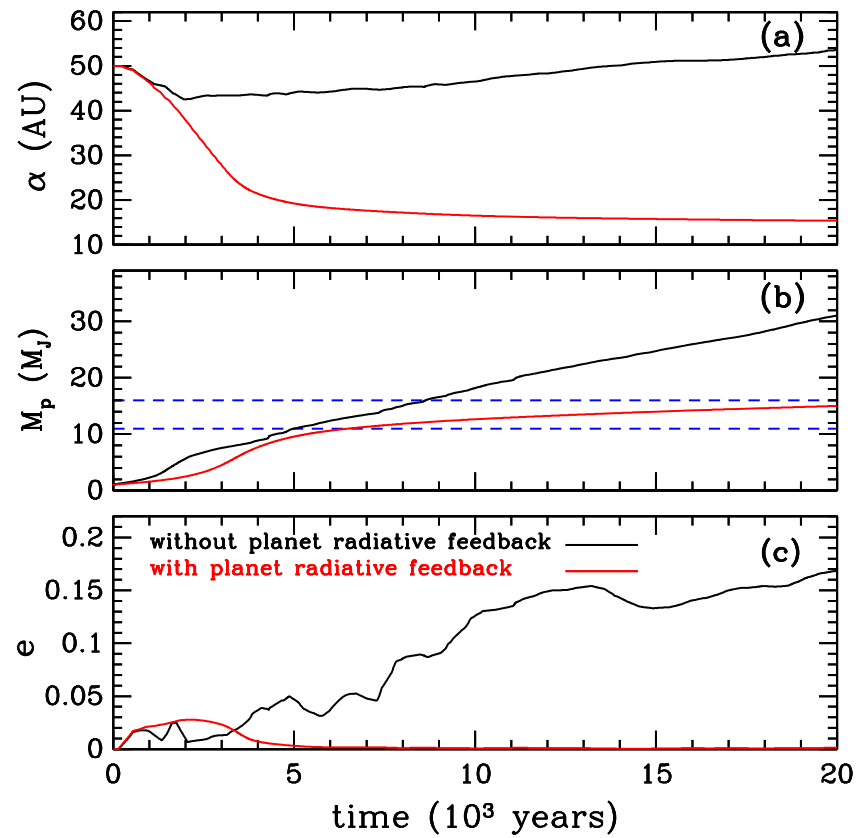

Figure 2. Evolution of (a) the semi-major axis $\alpha$, (b) the mass $M_{\mathrm{p}}$, and (c) the eccentricity $e$ of the protoplanet. The protoplanet initially migrates quickly inward, but it grows in mass and it is able to open up a gap in the disk. The migration then is essentially halted. In the non-radiative case the protoplanet becomes a brown dwarf, whereas in the case with radiative feedback mass growth is suppressed and the protoplanet's mass remains within the planetarymass regime (marked by the horizontal dashed lines in (b)). Radiative feedback keeps the protoplanet on a circular orbit $(e \approx 0)$.

accretion happens onto the second hydrostatic core. The radius of the second core is uncertain; it is estimated to be $\sim 1-20 R_{\odot}$ (Masunaga \& Inutsuka 2000; Tomida et al. 2013; Vaytet et al. 2013). Here we shall assume $R_{\text {acc }}=1 R_{\odot}$. The total pseudo-ambient temperature is

$$
T_{\mathrm{A}}^{4}(\boldsymbol{r})=\left[T_{\mathrm{A}}^{\star}(\boldsymbol{r})\right]^{4}+\left[T_{\mathrm{A}}^{\text {planet }}(\boldsymbol{r})\right]^{4}
$$

We note that the radiative feedback from the star is fixed, whereas the radiative feedback from the protoplanet is variable with time and depends on the accretion of gas onto it.

\section{MIGRATION OF GAS GIANT PLANETS IN GRAVITATIONALLY UNSTABLE DISKS}

We investigate the planet-disk interactions and the evolution of the properties of the protoplanet in two cases: with and without radiative feedback from the protoplanet (Figure 1). The initial evolution of the protoplanet orbital parameters and mass shows a similar pattern in both cases (Figure 2). The protoplanet initially migrates inward quickly, with a migration timescale of $\sim 10^{4}$ years, in agreement with previous studies (Baruteau et al. 2011; Michael et al. 2011). As the protoplanet migrates inward, its mass increases significantly by accreting gas from the disk. Eventually the protoplanet is massive enough to be able to open up a gap and the migration either slows down (when the radiative feedback from the protoplanet is taken into account; the migration timescale is $\sim 10^{5}$ years) or even changes to a slight outward migration (for the case without radiative feedback from the protoplanet; the outward migration timescale is $\sim 10^{5}$ years). After the gap opens up, the protoplanet continues to grow in mass but rather slowly, mainly
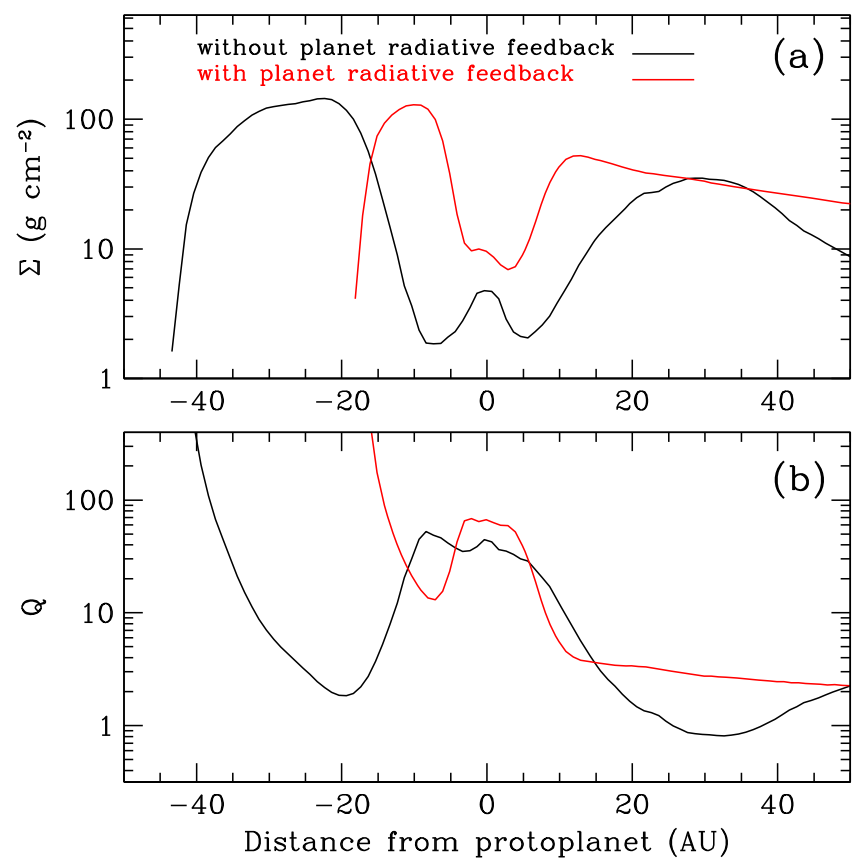

Figure 3. (a): Azimuthally averaged surface density of the disk in the protoplanet neighborhood (at $5 \mathrm{kyr}$ ), for the two cases examined here (without/ with irradiation from the protoplanet). The radial cells where the surface density is averaged are centered around the star, but the distance in the graph is given with respect to the cell of the protoplanet (to make comparison easier). The gap is shallower and narrower when radiative feedback from the protoplanet is taken into account. (b): Same as above, but for the azimuthally averaged Toomre parameter $Q(R)=c(R) \Omega(R) / \pi G \Sigma(R)$, where $c$ is the midplane isothermal sound speed, $\Omega$ is the the angular velocity, $\Sigma$ is the surface density, and $R$ is the distance from the star. Radiative feedback from the protoplanet stabilizes the disk $(Q>1)$.

by accreting material from its circumplanetary disk, a disk formed within the Hill sphere of the protoplanet. The time required for the opening up of a gap is different between the two cases that we present here. In the radiative feedback case the radiation emitted from the protoplanet heats the disk, the opening up of the gap is more difficult and takes twice as much time to happen $\left(4 \times 10^{4}\right.$ years in comparison with $2 \times 10^{4}$ years for the non-radiative protoplanet). The gap not only takes more time to open but it is also shallower and less wide (Figure 3(a)) as the protoplanet moves closer to the central star (where both the disk-scale height and Hill radius of the protoplanet are smaller). The protoplanet grows in mass at a slower rate, and the inward migration continues. The protoplanet in this case migrates closer to the central star (Figure 2(a)) but its mass growth is considerably suppressed (Figure 2(b)). At the same time, the orbit of the protoplanet is circularized (Figure 2(c)).

The final outcome of disk-planet interactions is distinctly different for the two cases presented here (Figure 1). In the case without radiative feedback the protoplanet eventually becomes a brown dwarf with a mass of $28 M_{\mathrm{J}}$, on a eccentric orbit $(e \approx 0.17)$, and a semi-major axis of $50 \mathrm{AU}$. When irradiation from the accreting protoplanet is included in the model, the protoplanet reaches a mass of only $14 M_{\mathrm{J}}$, i.e., around the deuterium burning limit and within the planetary-mass regime (<11-16.3 $M_{\mathrm{J}}$; Spiegel et al. 2011), while it remains on a relatively wide (semi-major axis of $15 \mathrm{AU}$ ), circular orbit, avoiding excessive inward migration. 
The role of the radiative feedback from the protoplanet is critical. Initially, irradiation from the protoplanet heats the disk, making the opening up of the gap more difficult, while thermal pressure delays large gas accretion onto the protoplanet. Therefore, the protoplanet continues its inward migration without accreting excessively. Its migration is eventually slowed down once the gap is opened up but the protoplanet still continues to heat and stabilize the disk. The effective viscosity due to the disk self-gravity is small and the inward flow of gas is relatively slow. Most of the gas within the protoplanet orbital radius is accreted onto the central star, creating an inner hole in the disk. On the other hand, when radiative feedback from the protoplanet is not taken into account, the disk remains unstable; the Toomre parameter $\mathrm{Q}$ at the edges of the gap is below 1 (see Figure 3(b)). Therefore, the gas flows rather quickly toward the outer edge of the gap, onto the circumplanetary disk and eventually onto the protoplanet, as the effective viscosity due to the disk self-gravity is large. The gravitationally unstable gap edges may also be responsible for the outward migration of the protoplanet (Lin \& Papaloizou 2012).

These results contradict previous findings in which inward migration is fast and continues toward the central star as the protoplanet is not able to open up a gap (Baruteau et al. 2011; Michael et al. 2011), questioning whether the survival of giant planets formed early on during the lifetime of a disk by fragmentation is possible. The critical difference between this work and previous models is that the protoplanet is allowed to grow in mass and therefore becomes massive enough to be able to open up a gap and its migration is slowed down.

\section{NUMERICAL RESOLUTION AND COMPARISON WITH PREVIOUS STUDIES}

To test our numerical code we performed simulations of disk-planet interactions in a system where a Jupiter-mass planet is embedded in a low-mass $\left(0.005 M_{\odot}\right)$ disk at a circular orbit at a distance of $5.2 \mathrm{AU}$ from a $1-M_{\odot}$ star. This problem has been studied both numerically, with grid-based (Bate et al. 2003) and particle-based (Ayliffe \& Bate 2009) codes, and analytically (Ward 1997). We performed simulations matching the initial conditions of previous authors (Ayliffe \& Bate 2009) using $10^{6}$ and $2 \times 10^{6} \mathrm{SPH}$ particles. The Hill radius of the planet is resolved adequately: the radius of the planet sink is set to $0.1 R_{\mathrm{H}}$ (where $R_{\mathrm{H}}$ is the Hill radius of the planet), while the number of SPH particles that is used ensures that the smoothing length, which defines the spatial resolution in SPH simulations at the planet's initial position, is $0.17 R_{\mathrm{H}}$ and $0.13 R_{\mathrm{H}}$ for the simulations with $10^{6}$ and $2 \times 10^{6}$ particles, respectively. We find that the planet migrates inward as expected, with a migration timescale of (1.7-2.6) $\times 10^{4}$ year when radiative transfer is included (compared to $1.5 \times 10^{4}$ year in the literature; Ayliffe \& Bate 2009), and (4-7) $\times 10^{4}$ year for the local isothermal case, i.e., where the disk temperature depends only on the distance from the central star (compared to $(9-11) \times 10^{4}$ year and $(6-12) \times 10^{4}$ year in the literature; Bate et al. 2003; Ayliffe \& Bate 2009). Therefore our calculations are in very good agreement with previous estimates of migration timescales.

In the case of the massive, wide-orbit planets that we examine here, the planet's Hill radius is much larger and therefore easier to resolve. For example, when the Jovian protoplanet is at $50 \mathrm{AU}$ away from the central star its Hill

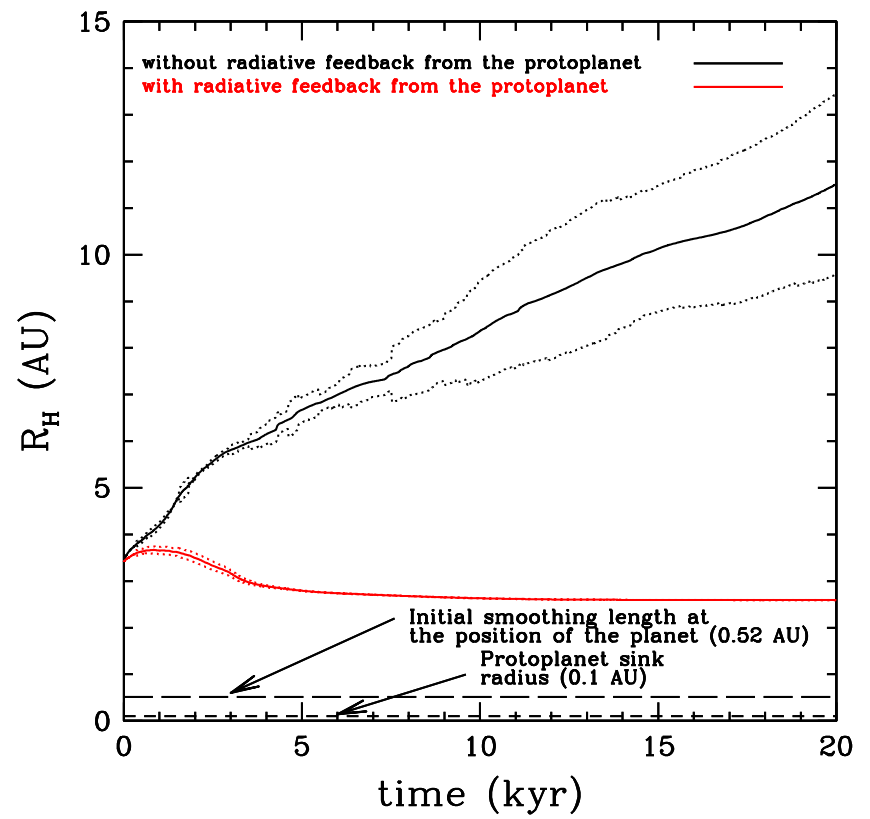

Figure 4. Evolution of the Hill radius, $R_{\mathrm{H}}$, of the protoplanet with time, compared with the smoothing length $(0.52 \mathrm{AU})$, which defines the spatial resolution in SPH simulations, at the planet's initial position, and the protoplanet sink radius $(0.1 \mathrm{AU})$. The Hill sphere of the protoplanet is resolved adequately.

radius is $R_{\mathrm{H}} \sim 3.5 \mathrm{AU}$. We use $10^{6} \mathrm{SPH}$ particles so that the smoothing length at the initial position of the protoplanet is $0.1 \mathrm{AU}$, i.e., just $0.03 R_{\mathrm{H}}$. This value remains much smaller than the Hill radius, as this value changes while the planet accretes mass and migrates within the disk (Figure 4). Another validation for our computational method comes from the fact that the migration timescale that we obtain for the initial stage of planet migration (before a gap is opened up) is $10^{4}$ years, which is in excellent agreement with previous studies (Baruteau et al. 2011; Michael et al. 2011) using completely different types of codes: FARGo (Baruteau et al. 2011) and CHYMERA (Michael et al. 2011).

\section{DISCUSSION}

This work demonstrates the combined effect of gas accretion onto a protoplanet and the associated accretion-powered radiative feedback from the protoplanet, on regulating its migration and mass growth. Previous studies have shown that radiative heating from an Earth-like protoplanet forming in a low-mass disk may in fact stop its inward migration or even reverse it (Benítez-Llambay et al. 2015). It has also been suggested that radiative feedback may delay the contraction of the protoplanetary core so that it is tidally destroyed as it moves closer to the central star (Nayakshin \& Cha 2013). The amount of energy radiated from the protoplanet depends on where the accretion happens, i.e., the radius of the protoplanet, which is uncertain. It has been suggested that this energy that is released heats the region around the protoplanet, providing a way to infer the presence of a protoplanet in a disk (Montesinos et al. 2015).

Here we show that accretion and radiative feedback work in opposing ways: gas accretion increases the mass of the protoplanet and supports the creation of a gap in the disk, whereas radiative feedback, which is the result of gas accretion, works to suppress accretion onto the protoplanet and inhibit/ 
delay gap opening by increasing the disk temperature. It heats and stabilizes the disk, suppressing fast gas accretion. Higher gas accretion induces higher radiative feedback that in turn opposes further gas accretion. Therefore, accretion and feedback are self-regulated and the final outcome of their combined effect is an inward migration of the protoplanet with a more moderate increase of its mass than in the case without radiative feedback. Eventually the protoplanet is able to open up a gap, the migration is effectively halted, and the protoplanet survives on a wide orbit.

The final mass of the protoplanet in the simulation with radiative feedback is around the deuterium burning limit. This simulation covers only a period of $20 \mathrm{kyr}$, thus if gas accretion continues, the mass of the protoplanet could further increase. However, radiative feedback may delay gas accretion until other disk dispersal mechanisms (e.g., photoevaporation) come into play.

The ramifications of these processes are important for the formation of giant planets on wide orbits. Only a few planets of this type have been observed so far, but this is likely due to observational biases, as these are faint objects next to bright stars and both high sensitivity and high-angular resolution observations are essential. However, more wide-orbit giant planets are bound to be discovered with new surveys using specialized observing techniques and instruments such as the Gemini Planet Imager (Macintosh et al. 2014), SPHERE/VLT (Beuzit et al. 2008), and HiCIAO/SUBARU (Suzuki et al. 2009)). Therefore, it is critical to explain their formation mechanisms and determine their connection to the formation of planetary systems and the formation of our own solar system.

Here we have shown that if massive protoplanets form by the gravitational fragmentation of disks, gas accretion and radiative feedback regulate their mass growth and inward migration so that they are able to avoid excessive mass growth and rapid inward migration; their masses remain around the deuterium burning limit for at least the initial stages of their evolution, they avoid disruption, and they survive on almost circular wide orbits around Sun-like stars. Therefore, giant planets like the ones in the HR 8799 system may have formed by disk fragmentation. Planet formation by gravitational fragmentation of protostellar disks may only happen during the early stages in a disk's lifetime, on a timescale of a few thousand years, while disks are still massive enough to be prone to gravitational instabilities. The presence of a massive giant planet within the disk plays an important role in the disk's evolution and affects the dynamics of dust particles as they coagulate to form larger bodies and eventually planets within a few million years. Therefore, the formation of massive gas giant planets at an early stage and their survival on wide orbits sets the disk environment in which the subsequent formation of terrestrial planets, like Earth, happens.

I thank S. Inutsuka, H. Kobayashi, S. Ida, Y. Fujii, and M. Kunitomo for stimulating discussions on the work presented here. I also thank the anonymous referee for useful comments. Simulations were performed using the UCLAN HPC Cluster
WILDCAT. The column density plots were produced using SPLASH (Price 2007). I acknowledge support from STFC grant ST/ M000877/1 and from a Royal Society-Daiwa Foundation International Exchanges award.

\section{REFERENCES}

Aller, K. M., Kraus, A. L., Liu, M. C., et al. 2013, ApJ, 773, 63

Andrews, S. M., Czekala, I., Wilner, D. J., et al. 2009, ApJ, 700, 1052

Ayliffe, B. A., \& Bate, M. R. 2009, MNRAS, 393, 49

Bailey, V., Meshkat, T., Reiter, M., et al. 2014, ApJL, 780, L4

Baruteau, C., Meru, F., \& Paardekooper, S.-J. 2011, MNRAS, 416, 1971

Bate, M. R., Lubow, S. H., Ogilvie, G. I., \& Miller, K. A. 2003, MNRAS, 341,213

Benítez-Llambay, P., Masset, F., Koenigsberger, G., \& Szulágyi, J. 2015, Natur, 520, 63

Beuzit, J.-L., Feldt, M., Dohlen, K., et al. 2008, Proc. SPIE, 7014, 701418

Boley, A. C. 2009, ApJL, 695, L53

Boley, A. C., Hayfield, T., Mayer, L., \& Durisen, R. H. 2010, Icar, 207, 509

Boss, A. P. 1997, Sci, 276, 1836

Cameron, A. G. W. 1978, M\&P, 18, 5

Forgan, D., Rice, K., Stamatellos, D., \& Whitworth, A. P. 2009, MNRAS, 394, 882

Galicher, R., Rameau, J., Bonnfoy, M., et al. 2014, A\&A, 565, L4

Goldreich, P., \& Ward, W. R. 1973, ApJ, 183, 1051

Hubber, D. A., Batty, C. P., Mcleod, A., \& Whitworth, A. P. 2011, A\&A, 529, A27

Ireland, M. J., Kraus, A. L., Martinache, F., Law, N., \& Hillenbrand, L. A. 2011, ApJ, 726, 113

Kratter, K. M., Murray-Clay, R. A., \& Youdin, A. N. 2010, ApJ, 710, 1375

Kraus, A. L., Ireland, M. J., Cieza, L. A., et al. 2014, ApJ, 781, 20

Kuiper, G. P. 1951, PNAS, 37, 1

Kuzuhara, M., Tamura, M., Kudo, T., et al. 2013, ApJ, 774, 11

Lin, M.-K., \& Papaloizou, J. C. B. 2012, MNRAS, 421, 780

Low, C., \& Lynden-Bell, D. 1976, MNRAS, 176, 367

Machida, M. N., Inutsuka, S.-i., \& Matsumoto, T. 2006, ApJL, 649, L129

Macintosh, B., Graham, J. R., Ingraham, P., et al. 2014, PNAS, 111, 12661

Marois, C., Macintosh, B., Barman, T., et al. 2008, Sci, 322, 1348

Masunaga, H., \& Inutsuka, S. 2000, ApJ, 531, 350

Michael, S., Durisen, R. H., \& Boley, A. C. 2011, ApJL, 737, L42

Mizuno, H. 1980, PThPh, 64, 544

Montesinos, M., Cuadra, J., Perez, S., Baruteau, C., \& Casassus, S. 2015, ApJ, 806,253

Nayakshin, S., \& Cha, S.-H. 2013, MNRAS, 435, 2099

Pollack, J. B., Hubickyj, O., Bodenheimer, P., et al. 1996, Icar, 124, 62

Price, D. J. 2007, PASA, 24, 159

Rafikov, R. R. 2005, ApJL, 621, L69

Rameau, J., Chauvin, G., Lagrange, A.-M., et al. 2013, A\&A, 553, A60

Safronov, V. S., \& Zvjagina, E. V. 1969, Icar, 10, 109

Semenov, D., Henning, T., Helling, C., Ilgner, M., \& Sedlmayr, E. 2003, A\&A, 410, 611

Spiegel, D. S., Burrows, A., \& Milsom, J. A. 2011, ApJ, 727, 57

Stamatellos, D., \& Whitworth, A. P. 2009a, MNRAS, 392, 413

Stamatellos, D., \& Whitworth, A. P. 2009b, MNRAS, 400, 1563

Stamatellos, D., Whitworth, A. P., Bisbas, T., \& Goodwin, S. 2007, A\&A, 475,37

Stamatellos, D., Whitworth, A. P., \& Hubber, D. A. 2011, ApJ, 730, 32

Stamatellos, D., Whitworth, A. P., \& Hubber, D. A. 2012, MNRAS, 427, 1182

Suzuki, R., Tamura, M., Suto, H., et al. 2009, AIP Conf. Ser. 1158, Exoplanets and Disks: Their Formation and Diversity, ed. T. Usuda, M. Tamura \& M. Ishii (Melville, NY: AIP), 293

Tomida, K., Tomisaka, K., Matsumoto, T., et al. 2013, ApJ, 763, 6

Tsukamoto, Y., Machida, M. N., \& Inutsuka, S.-i. 2013, MNRAS, 436, 1667

Vaytet, N., Chabrier, G., Audit, E., et al. 2013, A\&A, 557, A90

Ward, W. R. 1997, Icar, 126, 261

Whitworth, A. P., \& Stamatellos, D. 2006, A\&A, 458, 817

Zhu, Z., Hartmann, L., Nelson, R. P., \& Gammie, C. F. 2012, ApJ, 746, 110 